\section{Measurements of linear dimensions on fundus photographs: comparison between photographic film and digital systems}

\author{
Abstract \\ Purpose The aim of this study is to compare \\ linear fundal landmarks measurements of \\ standard photographic film with colour digital \\ fundal images. \\ Methods Pairs of fundus fluorescein images \\ were acquired with a digital system and \\ photographic film on 10 patients. Each pair of \\ images was assessed independently by two \\ observers who calculated the distance between \\ two selected points on each image using easily \\ identifiable landmarks. Measurements made \\ using film images were compared to \\ measurements made using the corresponding \\ digital images. Agreement between the two \\ methods of measurements was assessed using \\ a linear correlation and graphical method. \\ Results The patients were divided into two \\ groups based on the field setting on the digital \\ software. In Group 1, the correlation \\ coefficient was 0.99 and in Group 2 it was 0.98 , \\ indicating a high level of agreement between \\ measurements with the computer measuring \\ tool for digital images compared to \\ photographic films using a standard \\ reticule. \\ Conclusion On the basis of the strong \\ correlation between linear fundal landmark \\ measurements between standard photographic \\ film and colour digital fundal images, we \\ conclude that the measurements from the \\ digital images are acceptable for diagnostic \\ and therapeutic purposes. Although there \\ was a tendency for the measurements to be \\ less accurate with increasing distances \\ measured. \\ Eye (2003) 17, 619-622. doi:10.1038/ \\ sj.eye. 6700418
}

M Musadiq, E Patsoura, S Hughes and YC Yang

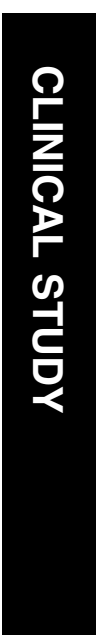

Keywords: fundus photography; linear measurements; digital measuring tool

Introduction

Quantitative analysis of fundal lesions is important in many areas of ophthalmology including the assessment of lesion size for laser therapy in photodynamic therapy and also for the monitoring of potentially progressive lesions like choroidal naevi. Digital acquisition systems are increasingly employed in fundus photography. The instant availability of the images on the computer monitor allows instant interpretation leading to earlier diagnosis of fundal abnormalities. Many digital systems offer a built-in measuring tool allowing quantitative analysis of linear and spatial dimensions of posterior pole features. We are not aware of any study that has evaluated the reliability of such measuring tools on a digital system. In this study, we investigated the measuring tool in our digital system by comparison with a standard technique employed in the analogue film system.

\section{Materials and methods}

A Topcon TR501A fundus camera equipped with a high-resolution Kodak Megaplus monochrome digital camera as well as a conventional film camera back was used. ${ }^{1}$ The digital system used was Topcon Imagenet 2000. During routine fluorescein angiography studies on 10 patients, with refractive errors of less than $4 \mathrm{D}$, images were captured using the digital system as well as film. All images were captured with a $35^{\circ}$ field setting on the camera. This produces the same magnified view both on
Wolverhampton and Midlands Counties Eye Infirmary

West Midlands, UK

Correspondence:

M Musadiq

Wolverhampton and

Midlands Counties

Eye Infirmary

Compton Road

Wolverhampton WV3 9QR

West Midlands, UK

Tel: + 44902307999

Fax: + 44902645018

E-mail: mmusadiq@

hotmail.com

Received: 8 July 2002 Accepted in revised form: 27 August 2002 
film as well as on the monitor. However, in our model of Topcon camera, the field setting on the capture software has to be manually selected. This gives an opportunity to test the accuracy of the measuring tool if the field setting on the software did not correspond to that of the camera.

In the first five patients (group 1), the field settings on the camera and in the capture software were set at $35^{\circ}$. In the next five patients (group 2), the settings were mismatched so that the camera was set at $35^{\circ}$ while the capture software was set at $50^{\circ}$. In total, 10 film images, each with a corresponding digital image from 10 patients, were used for the study. In each image, 10 wideranging linear dimensions were selected using easily identifiable landmarks. ${ }^{2}$ For each linear dimension chosen, the distance was measured on the film from the negative using a transparent reticule with $0.1 \mathrm{~mm}$ intervals and divided by 2.5 to correct for the camera's magnification. ${ }^{3}$ The corresponding linear dimension on the digital image was measured using the software's measuring tool. Measurements taken with the two methods were compared using linear correlation as well as a graphical method for agreement and bias.,

\section{Results}

A total of $10 \mathrm{film} /$ digital pairs of images were used for comparison. In each pair, 10 linear dimensions were chosen giving a total of 100 comparisons. The magnitude of the dimensions selected ranged from 0.6 to $10.4 \mathrm{~mm}$.

In group 1, where the camera's field setting and the capture software's setting were $35^{\circ}$, the correlation between measurements obtained on film and those obtained digitally are shown in Figure 1 . The correlation coefficient was 0.99 indicating a high correlation between the two methods of measurement. Agreement between analogue measurements and digital measurements is shown in Figure 2. On average, the film measurements were $0.32 \mathrm{~mm}$ less than digital. The 2SD limits of agreement were $(-0.88$ to +0.24$)$, indicating high level of agreement between analogue and digital. However, it is clear from the agreement plot in Figure 2 that agreement between the two methods decreased with increasing dimensions.

In group 2, where there was a discrepancy between the settings of the fundus and the digital camera $\left(35^{\circ}\right.$ for the fundus and $50^{\circ}$ for the digital), the correlation coefficient was also high (0.98), but from the agreement plot (Figure 3) analogue measurements were $1.25 \mathrm{~mm}$ less than digital measurements on average. The 2SD limits of agreement were -2.91 to $+0.40 \mathrm{~mm}$. The incorrect setting gave rise to considerable bias in the digital measurements. There was also a tendency for the measurements to be less accurate with increasing distances measured.

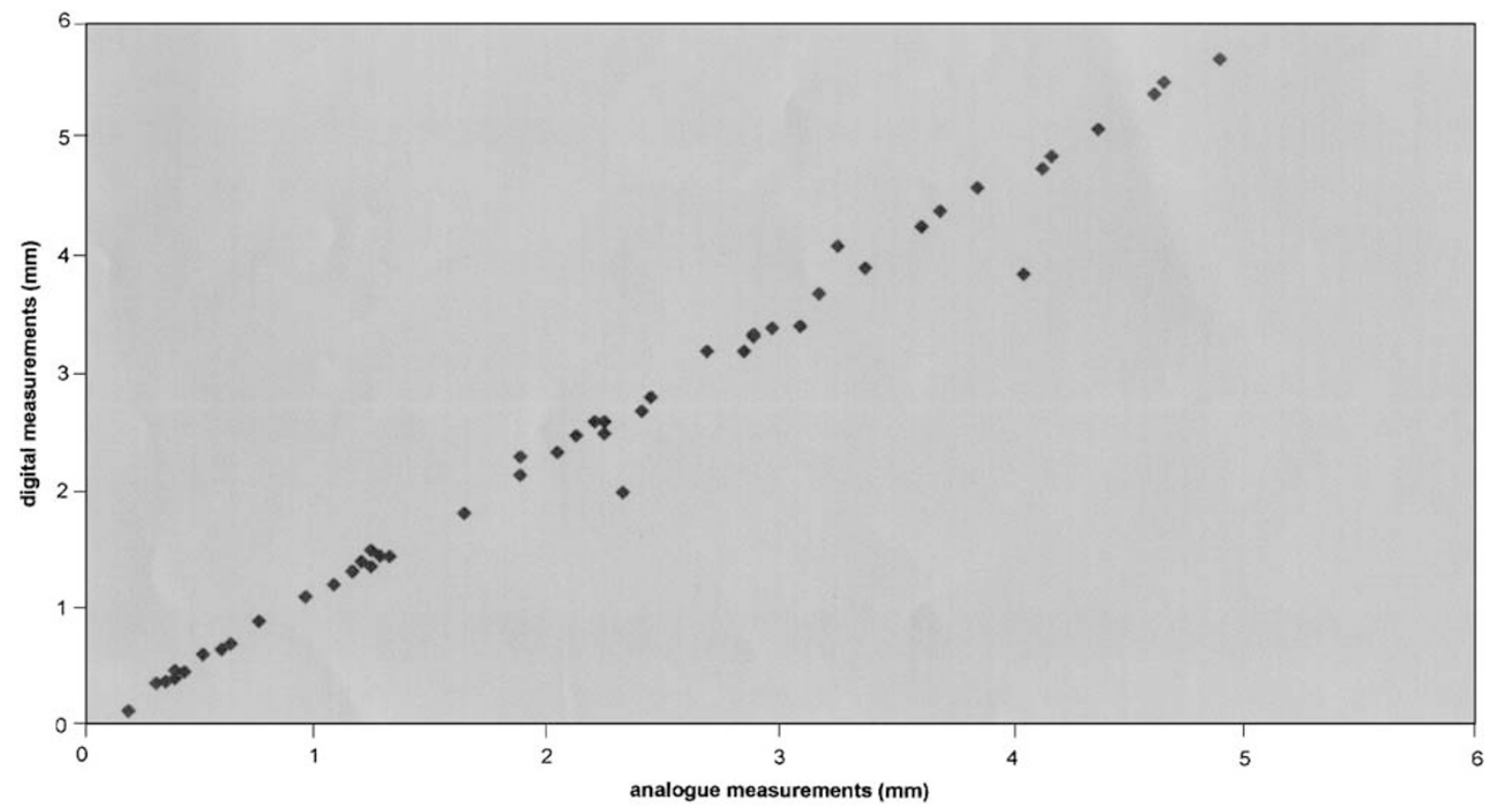

Figure 1 Group 1-correlation measurements. 


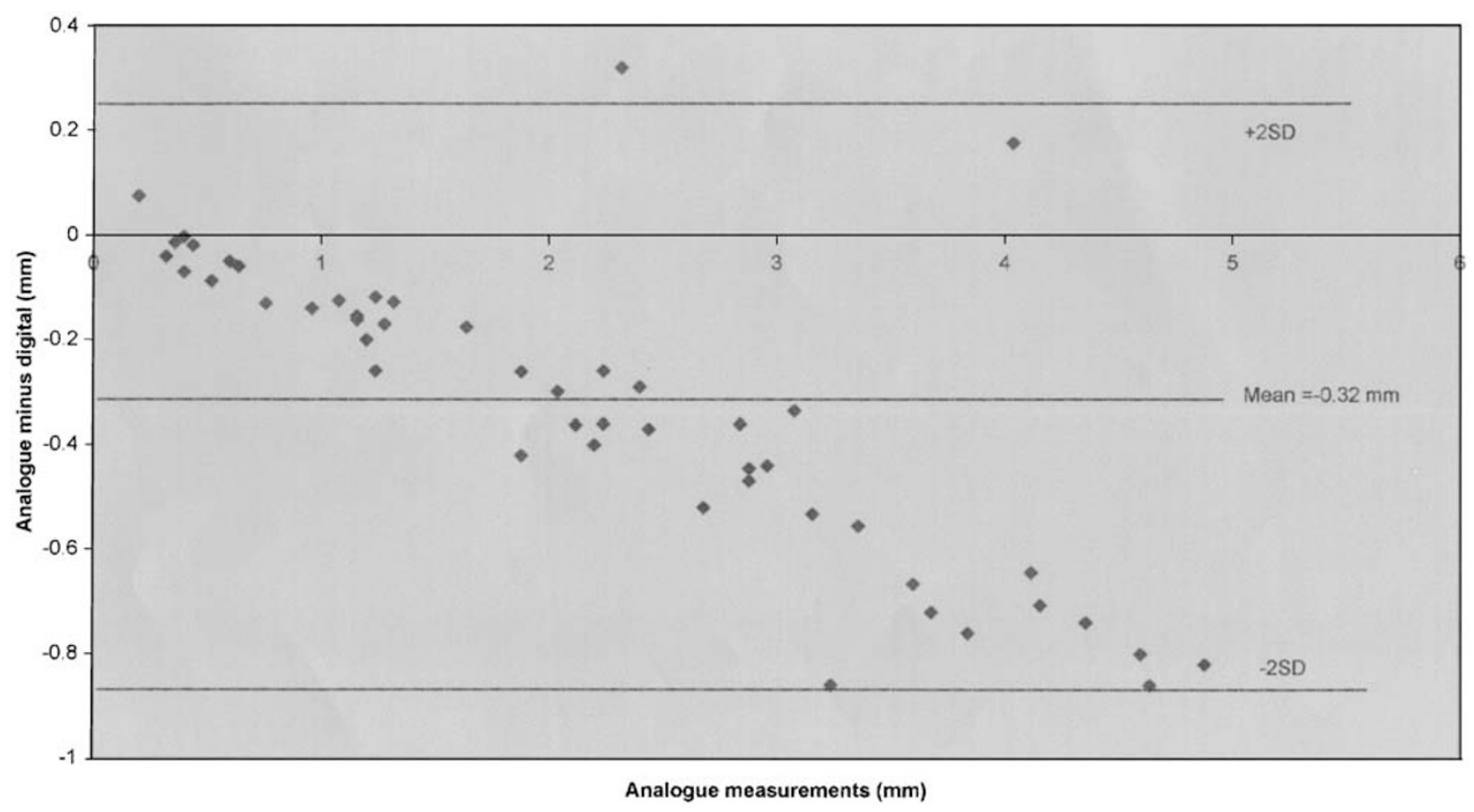

Figure 2 Group 1-agreement between analogue and digital measurements.

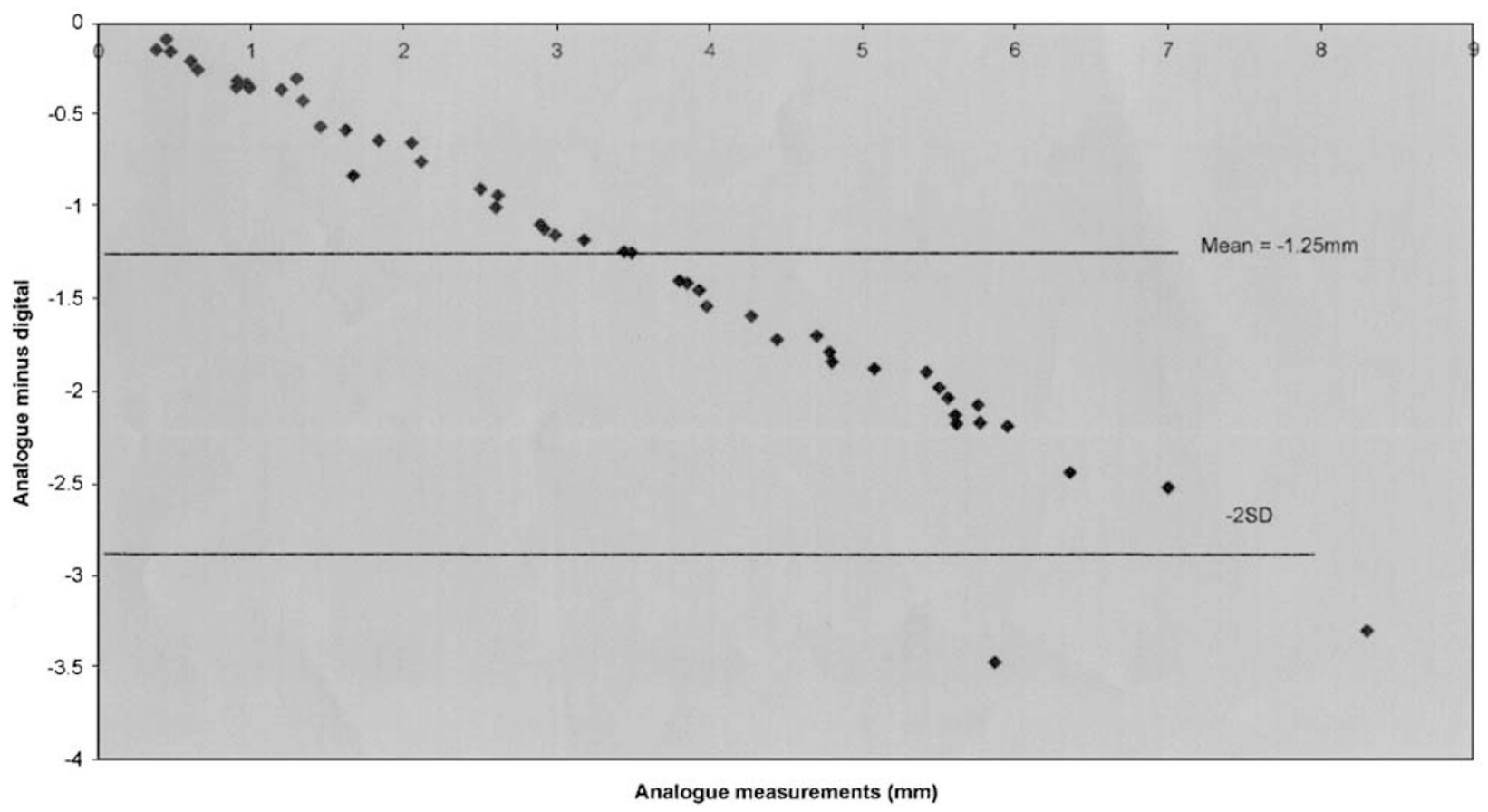

Figure 3 Group 2-agreement between analogue and digital measurements.

\section{Discussion}

Linear dimensions measured using the measuring tool on the Imagenet 2000 software showed a good level of agreement with those estimated using the conventional reticule on film negative method and should be reliable when used in clinical practice. Although larger dimensions were associated with less agreement, the 
deviations were small as shown by the 2SD limits of within $1 \mathrm{~mm}$.

In this study, we compared images taken by the same camera using different capturing media so the refractive errors of the patients were not relevant. To ensure good image quality, we used patients with low refractive errors. ${ }^{6,7}$

The graphical method of assessing agreement used in this study merely compared the digital system to a standard analogue technique. It does not imply that the analogue technique is more accurate than the digital system. It is possible that there could have been some operator error involved in either technique. Without resorting to doing a repeatability type evaluation of what was essentially a length measuring procedure, the high level of correlation (0.99) between the two types of measurements indicates that there is unlikely to be any significant random operatorerror as a lower correlation coefficient would be obtained.

From the agreement plots, the bias was negligible when the capture software was set correctly at $35^{\circ}$. However, in group 2 when the capture software was set incorrectly to $50^{\circ}$, a significant bias in the measurement was introduced. This problem can occur in older cameras such as the one used in our study. When using such a camera, the field of view shown on the monitor screen of the image captured corresponds to the field setting on the camera and not to the field setting on the capture software. The measuring tool calibration adopted by the software depends on the field setting on the software at the time of capture. It is an easy pitfall to capture at the wrong field setting, which could give rise to erroneous measurements. Fortunately, with many newer camera models and newer imaging software, when the field setting is altered on the camera, the connectivity functions between the camera and the imaging software ensure that the software capture settings are altered automatically. This is an important feature to look for especially when using imaging software, which is not supplied by the camera manufacturer.

\section{References}

1 Mimoun G, Soubrane G, Coscas G. Clinical experience with digitised fluorescein angiography. Int Ophthalmol 1991; 15: $41-46$.

2 Iester M, Mochi B, Lai S, Rathschuler F, Rolando M, Ciurlo G. Intraimage reproducibility of measurements in the macular area using a computerized system. Int Ophthalmol 1997; 21: 153-159.

3 Bird AC, Bressler NM, Bressler SB, Chisholm IH, Coscas G, Davis MD. An international classification and grading system for age-related maculopathy and age-related macular degeneration. Survey Ophthalmol 1995; 395: 367-374.

4 Bland JM, Altman DG. Statistical methods for assessing agreement between two methods of clinical measurement. Lancet 1986; 307-310.

5 Lee J, Koh D, Ong CN. Statistical evaluation of agreement between two methods for measuring a quantitative variable Comput Biol Med 1989; 191: 61-70.

6 Arnold JV, Gates WC, Taylor KM. Possible errors in the measurement of retinal lesions. Invest Ophthamol Vis Sci 1993; 348: 2576-2580.

7 Rudnicka AR, Burk ROW, Habil M, Edgar DF, Fitzke FW. Magnification characteristics of fundal imaging systems. Ophthalmology 1998; 105: 2186-2192. 